\title{
Heterologous Expression of Salvia miltiorrhiza MicroRNA408 Enhances Tolerance to Salt Stress in Nicotiana benthamiana
}

\author{
Xiaorong Guo, Junfeng Niu * and Xiaoyan Cao *(D) \\ National Engineering Laboratory for Resource Development of Endangered Crude Drugs in Northwest of \\ China, Key Laboratory of the Ministry of Education for Medicinal Resources and Natural Pharmaceutical \\ Chemistry, Shanxi Normal University, Xi'an 710062, China; guoxiaorong@snnu.edu.cn \\ * Correspondence: niujunfeng@snnu.edu.cn (J.N.); caoxiaoyan@snnu.edu.cn (X.C.); \\ Tel.: +86-029-85310680 (J.N.); +86-029-85310260 (X.C.)
}

Received: 11 November 2018; Accepted: 7 December 2018; Published: 11 December 2018

\begin{abstract}
MicroRNAs (miRNAs) are a class of endogenous small RNAs that regulate the expression of target genes post-transcriptionally; they are known to play major roles in development and responses to abiotic stress. MicroRNA408 (miR408) is a conserved small RNA in plants; it was reported that miR408 genes were involved in abiotic stress in Arabidopsis. However, miR408 in Salvia miltiorrhiza has been rarely investigated. In this study, we cloned $S m-M I R 408$, the miR408 precursor sequence, and its promoter sequence from $S$. miltiorrhiza and the role in tolerance to salt stress is described. The effects of salt stress on miR408 expression were studied by using $\beta$-glucuronidase (GUS) staining. Our data indicated that transgenic tobacco overexpressing Sm-MIR408 promoted seed germination and reduced the accumulation of reactive oxygen species under salt stress. Transcript levels of antioxidative genes, i.e., $\mathrm{NbSOD}, \mathrm{NbPOD}$, and $\mathrm{NbCAT}$, and their enzyme activities increased in salinity-stressed transgenic tobacco plants, suggesting a better antioxidant system to cope the oxidative damage caused by salinity stress. Taken together, these findings indicated that miR408 functions in positive responses to salt tolerance in tobacco.
\end{abstract}

Keywords: abiotic stress; antioxidant enzyme; miR408; salt tolerance; Salvia miltiorrhiza

\section{Introduction}

Abiotic stresses such as salinity, drought, cold, and extreme temperature greatly affect the growth and development of plants. In order to cope with these challenges, plants have evolved complex molecular mechanisms to deal with unfavorable environments and to reduce the damage as much as possible, resulting in adaptive responses through physiological and morphological changes $[1,2]$. Increasing salinization of soil is one of the main factors influencing metabolic imbalance, excess accumulation of reactive oxygen species (ROS), reduction in photosynthetic performance, and nutrient absorption reduction [3,4]. Plants respond to the unfavorable environment and minimize possible damage by readjusting their physiological and biochemical levels. For example, $\mathrm{ROS}\left(\mathrm{H}_{2} \mathrm{O}_{2},-\mathrm{OH}\right)$ produced by aerobic metabolism can destroy cellular homeostasis and lead to cell death [5], and the ROS-scavenging system, which is mainly assisted by enzymatic systems, can reduce the impact of ROS on plants. Due to the unavoidable effects of abiotic stress on plants, it is critical to develop an effective and reliable procedure to mitigate the effects.

MicroRNAs (miRNAs) are a class of small non-coding RNAs 20-24 nucleotides in length, and they are derived from stem-loop precursors that repress target gene expression at the post-transcriptional level [6]. After integration into the RNA-induced silencing complex (RISC), miRNAs can form 
near-perfect pairs with their mRNA targets, based on sequence complementarity, and direct cleave the target genes or translational repression to play its biological function. As a new gene regulator, miRNAs have a similar function with the transcription factors $[7,8]$ which regulate development, growth and stress response in plants [9]. Various studies have demonstrated that miRNAs regulate the development and morphogenesis of leaves [10-12] and participate in the development of flowers [13-15]. Additionally, miRNAs respond to biotic and abiotic stresses like drought, salt, cold, and osmotic stress. For instance, drought stress induces miR159 [16] and miR319 [17] to accumulate in Arabidopsis, and the expression of miR156, miR159, and miR396 increased significantly under the condition of salt stress. Osmotic stress reduces the expression of miR398 and targets CSD1 and CSD2 that positively regulate oxidative stress tolerance in Arabidopsis, with miR398 as a positive regulator of oxidative stress responses [18].

MiR408 is a small RNA of 21 nucleotides, which was first discovered in Arabidopsis. At present, miR408 family comprises 56 members annotated in more than 30 plant species in miRBase database. The target genes of miR408 are blue copper protein members divided into two categories, including copper-binding proteins and laccase $[19,20]$. Targets of miR408 maintain copper homeostasis, and copper plays an important role in plants involved in photosynthesis, mitochondrial respiration, and lignification of the cell wall. Furthermore, the expression of miR408 is significantly influenced by various growth and environmental conditions. For example, miR408 is up-regulated in response to drought in Medicago truncatula [21] and Arabidopsis [22], while it is reduced in Oryza sativa [23] and Prunus persica [24]. Recent research has shown that miR408 is an important component of the HY5-SPL7 gene network that mediates the coordinated response to light and copper [25]. In Arabidopsis, overexpression of miR408 results in increased resistance to salinity, cold, and oxidative stress, but enhanced sensitivity to drought and osmotic stress [26]. Meanwhile, transgenic chickpea overexpressing miR408 showed increased drought tolerance [27]. Together, these results reveal that miR408 is involved in abiotic stress responses in plants, and it plays different roles in different species.

Salvia miltiorrhiza is one of the most commonly investigated medicinal plants, and its root is a traditional Chinese medicine for treating thoracic obstruction and heartache, amenorrhea, dysmenorrheal, and upset insomnia [28]. Now, it has been intensively studied for its protective role against cardiovascular diseases [29]. Although a few documents about microRNAs in S. miltiorrhiza have been reported [30,31], there is no report to date about functional study of microRNA from this species. In our previous work, we cloned a $366 \mathrm{bp}$ miR408 precursor sequence from S. miltiorrhiza and named it Sm-MIR408 [32]. Bioinformatics analysis showed that Sm-MIR408 can form a stable stem loop structure and it had a higher homology with tobacco and potato [31]. Tobacco belongs to the genus Nicotiana in the family Solanaceae, and is also readily transformable with a relatively short life cycle, which makes it a model plant for genetic studies.

To characterize the function of $S m-M I R 408$, we analyzed the functions of the 723-bp promoter region upstream of the predicted transcription initiation site of the Sm-MIR408-driven gene for $\beta$-glucuronidase (GUS), and Sm-MIR408 overexpressed in transgenic Nicotiana benthamiana, respectively. Our results showed that the expression of $S m-M I R 408$ is induced by salt treatment. Furthermore, overexpression of $S m-M I R 408$ in tobacco promoted seed germination under salt stress, and increased resistance to salt by activating the ROS-scavenging system. To our knowledge, this is the first report about a functional study of S. miltiorrhiza miRNA. Our data support that miR408 might serve as a potential target for genetic manipulations to engineer salt stress tolerance in diverse plants.

\section{Results}

\subsection{Sm-MIR408 Is Induced by Copper Deficiency and Salt Treatment}

It was observed in Arabidopsis that the abundance of miR408 is responsive to copper supply in the environment. The miR408 level was low with standard Murashige and Skoog (MS) media (with a copper concentration of $0.1 \mu \mathrm{m}$ ), but it increases markedly upon copper starvation [33]. Supplementing 
MS with $5 \mu \mathrm{m}$ copper effectively prevents the accumulation of miR408 [34]. Since miR408 is highly conserved in land plants, we speculate that Sm-MIR408 in S. miltiorrhiza probably responds to copper supply in a similar expression pattern to that in Arabidopsis. Here, we analyzed its expression when S. miltiorrhiza was grown on standard MS medium ( $0.1 \mu \mathrm{M}$ copper), MS medium-deficient copper $(0 \mu \mathrm{M}$, MS- $\mathrm{Cu}$ ) and sufficient copper $(5 \mu \mathrm{M}, \mathrm{MS}+\mathrm{Cu})$ conditions. Real-time quantitative PCR (RT-qPCR) results showed that the expression of $S m-M I R 408$ was strongly influenced by copper availability (Figure 1A). To further verify the expression of $S m-M I R 408$ in response to copper, transgenic tobacco expressing MIR408pro::GUS were cultured on MS, MS- $\mathrm{Cu}$ and MS+Cu medium. GUS staining revealed that the GUS signal was increased in transgenic tobacco grown on MS-Cu medium, and greatly decreased on MS+Cu medium, when compared with that grown on standard MS medium (Figure 1B). Our data indicated that transcription of $S m-M I R 408$ was strongly induced by copper deficiency, which was consistent with the results in Arabidopsis [33,34].

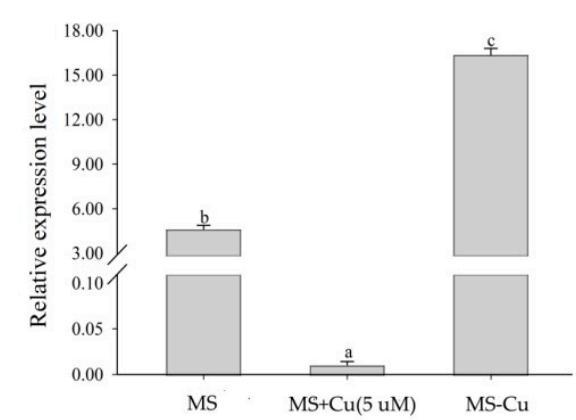

C

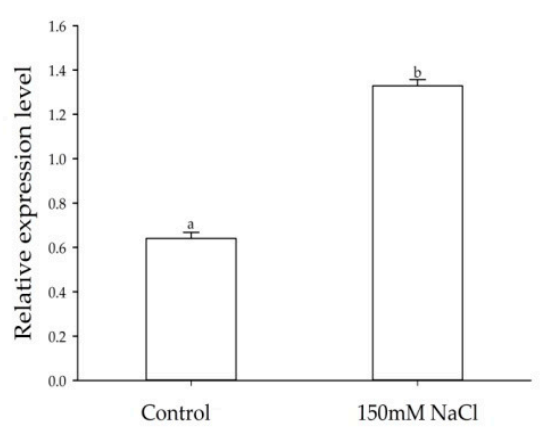

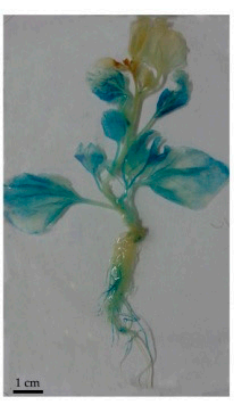

MS

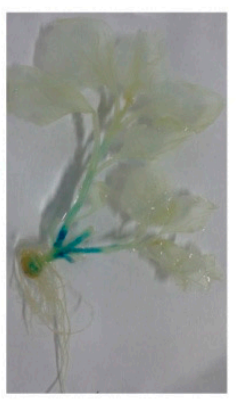

$\mathrm{MS}+\mathrm{Cu}(5 \mathrm{uM})$

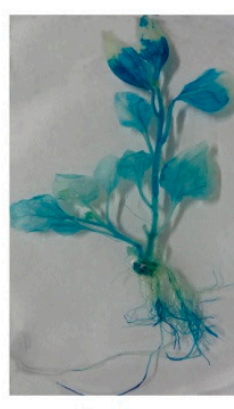

MS-Cu
$\mathrm{D}$
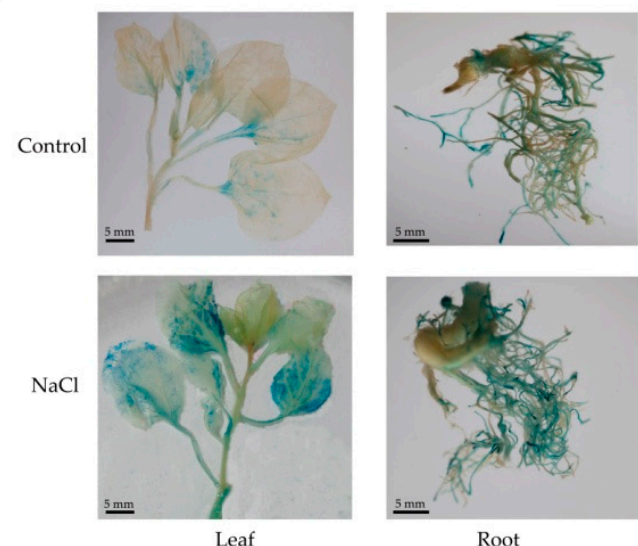

Figure 1. Transcription of $S m-M I R 408$ is induced under deficient copper conditions or $\mathrm{NaCl}$ treatment. (A) Expression level of Sm-MIR408 when two-week-old S. miltiorrhiza seedlings were transferred to standard Murashige and Skoog (MS) medium $(0.1 \mu \mathrm{M}$ copper $)$, MS medium deficient in copper $(0 \mu \mathrm{M}$, MS- $\mathrm{Cu}$ ) and sufficient copper $(5 \mu \mathrm{M}, \mathrm{MS}+\mathrm{Cu})$ for 7 days. (B) $\beta$-glucuronidase (GUS) staining results when one-week-old transgenic Nicotiana benthamiana expressing MIR408pro::GUS were transferred to MS, MS-Cu and MS+Cu for three weeks. (C) Expression levels of Sm-MIR408 in one-month-old Salvia miltiorrhiza seedlings treated with $150 \mathrm{mM} \mathrm{NaCl}$ for $24 \mathrm{~h}$. (D) The GUS staining in the transgenic Nicotiana benthamiana expressing MIR408pro::GUS after treatment with $150 \mathrm{mM} \mathrm{NaCl}$. For RT-qPCR, all data are means of three biological replicates, with error bars indicating SD; significant differences were determined using Duncan's multiple range test (indicated by different letters at $p<0.05$ ).

Since miR408 is involved in abiotic stress responses in plants [26,27], we further analyzed its expression under salt stress conditions. Real-time quantitative PCR (RT-qPCR) results showed that salt treatment significantly enhanced the expression of Sm-MIR408 in the seedlings of one-month-old S. miltiorrhiza (Figure 1C). Then, one-month-old T2 generation transgenic tobacco expressing 
MIR408pro::GUS were used to verify the expression of Sm-MIR408 in response to salt treatment. GUS staining revealed that the GUS signal was increased in both the leaves and roots of transgenic tobacco treated with $150 \mathrm{mM} \mathrm{NaCl}$ for $24 \mathrm{~h}$, compared to that of the control (Figure 1D). Altogether, these data suggest that the expression of $S m-M I R 408$ is up-regulated under salt stress condition.

\subsection{Heterologous Expression of Sm-MIR408 in Nicotiana benthamiana}

To evaluate the role of miR408 in the plant adaption to salt stress, we generated a Sm-MIR408 overexpression construct 35S::MIR408 driven by the cauliflower mosaic virus 35S (CaMV35S) promoter, which was introduced into the genome of tobacco through Agrobacterium tumefaciens-mediated transformation. To select positive transgenic plants overexpressing (OE) Sm-MIR408, we amplified the CaMV35S promoter by PCR with the genomic DNA of regenerated plants, and identified a total of six independent transgenic lines (Figure 2A). To verify the expression of Sm-MIR408 in transgenic tobacco, we conducted RT-qPCR to analyze the transcriptional levels of Sm-MIR408 in the transgenic lines. As demonstrated in Figure 2B, the transgenic lines showed different transcript abundances, while the transcripts of Sm-MIR408 was not detected in the wild-type (WT) control. To determine whether Sm-MIR408 can be successfully processed into mature miRNA, we conducted stem-loop RT-qPCR analyses and found that the expression level of mature miR408 in transgenic lines were significantly higher than that in the WT (Figure 2C), suggesting that the transcripts of Sm-MIR408 is properly processed into mature miR408.

A

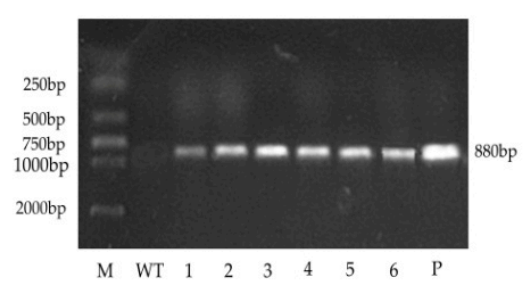

C

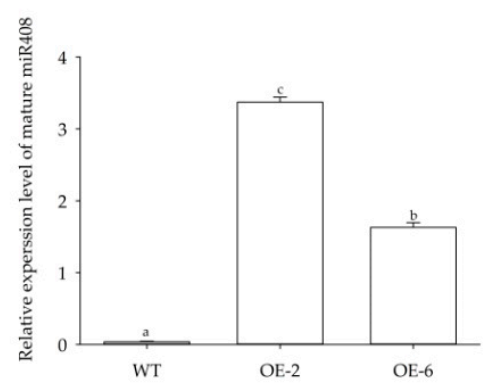

B

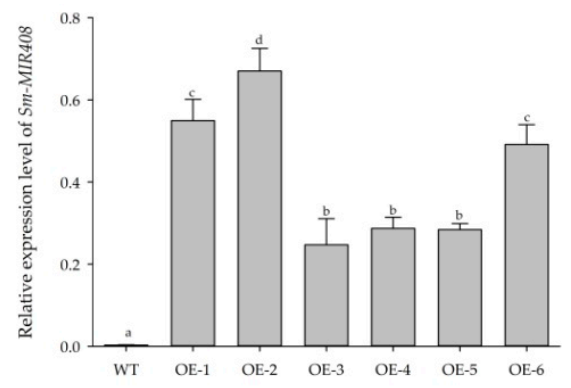

$\mathrm{D}$

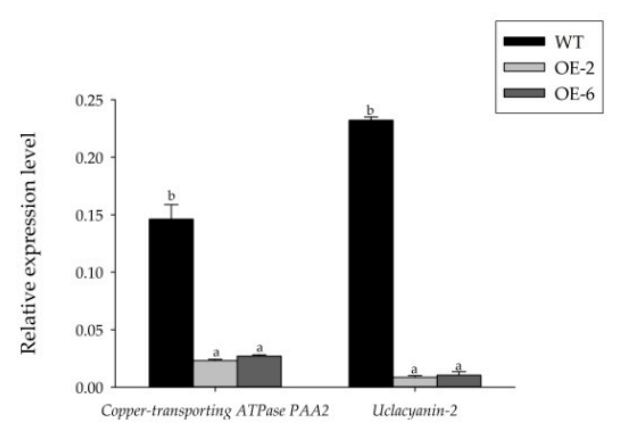

Figure 2. Detection of Sm-miR408 overexpressing (OE) transgenic lines. (A) PCR amplification product of $35 \mathrm{~S}$ promoter from transgenic Nicotiana benthamiana genomic DNA (gDNA). Lanes: M, DL2000 DNA marker; WT, wild-type plants as negative control; 1-6, different transgenic lines; P, plasmids as positive control. (B,C) Relative expression levels of Sm-MIR408 (B) and mature miR408 (C) in transgenic lines and WT. (D) Relative expression levels of copper-transporting ATPase PAA2 and uclacyanin-2 in transgenic lines and WT. The data represent means \pm SD of three independent experiments. Significant differences were determined using Duncan's multiple range test (indicated by different letters at $p<0.05$ ).

Based on the expression level of miR408, two transgenic lines, OE2 and OE6, were used in the following experiments. Since the targets of $\mathrm{Nb}$-miR408 were copper-transporting ATPase $P A A 2$ and uclacyanin-2 in N. benthamiana [35], we further analyzed their expression levels in the Sm-MIR408-overexpressing transgenic lines and WT by RT-qPCR. Our results indicated that the 
expression levels of copper-transporting ATPase PAA2 and uclacyanin-2 in transgenic lines were significantly lower than those in WT (Figure 2D).

\subsection{Sm-miR408 Confers Abiotic Stress Tolerance to Transgenic Plants}

To characterize the role that miR408 plays in abiotic stress tolerance, $\mathrm{T} 1$ transgenic lines OE2 and OE6 were selected for function analysis. The seeds of WT and transgenic lines were germinated on MS plates containing $150 \mathrm{mM} \mathrm{NaCl}$ to investigate seed germination and early seedling growth. Under normal growth condition, no significant difference was found in seed germination rates between WT and transgenic lines (Figure 3A). However, transgenic lines exhibited significantly higher germination rates than that of the WT on MS plates with $150 \mathrm{mM} \mathrm{NaCl}$ (Figure 3A). We further observed the growth phenotype of the transgenic lines and WT seedlings. As shown in Figure 3B,C, the transgenic lines and WT seedlings showed no significant phenotypic differences under normal conditions, while OE2 and OE6 exhibited improved root growth and significantly higher fresh weights than WT after cultivating for 21 days with salt treatment. The results indicated that overexpression of miR408 enhanced the tolerance to salt in tobacco.

A

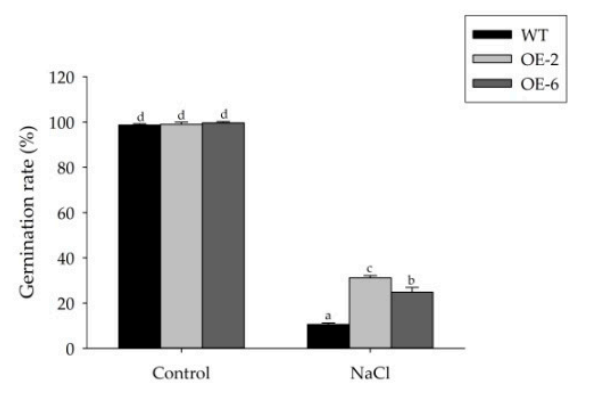

B

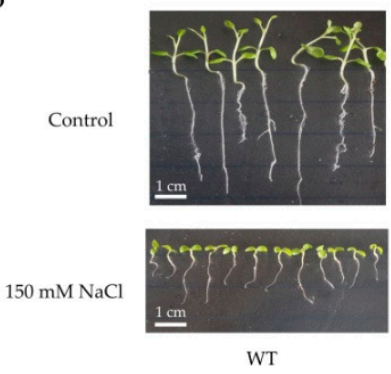

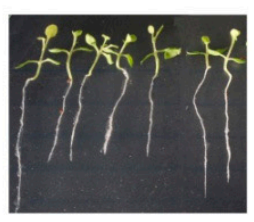

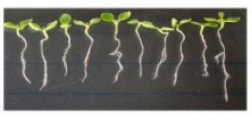

$\mathrm{OE}-2$
C
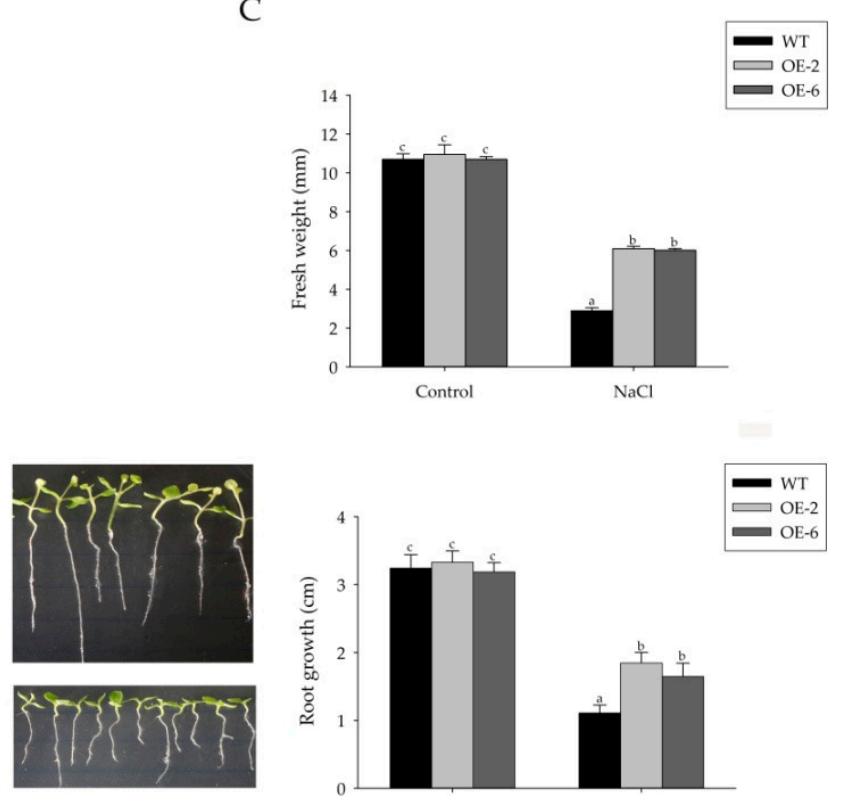

OE-6

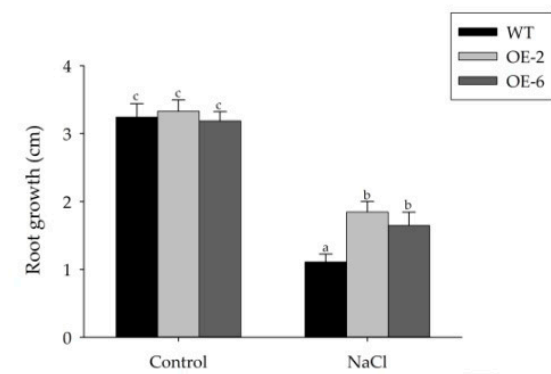

Figure 3. Salt stress tolerance assays of transgenic Nicotiana benthamiana. (A) Germination rates of wild type (WT) and miR408-overexpressing tobacco (OE2 and OE6) on MS medium containing $150 \mathrm{mM} \mathrm{NaCl}$ for 7 days. (B,C) The seedling phenotype (B) and statistical analysis of root length and fresh weight (C) when the seeds of transgenic lines (OE2 and OE6) and WT tobacco were cultured on MS medium and MS medium containing $150 \mathrm{mM} \mathrm{NaCl}$ for 21 days The error bars represent standard deviations of the mean measurements. Significant differences were determined using Duncan's multiple range test (indicated by different letters at $p<0.05$ ).

\subsection{Overexpression of Sm-miR408 Reduced ROS Accumulation under Salt Stress}

The abiotic stress leads to the generation of ROS, and the plants cope with excess ROS through its enhanced antioxidant defense system. Therefore, we examined the accumulation of hydrogen peroxide $\left(\mathrm{H}_{2} \mathrm{O}_{2}\right)$ and superoxide anion radical $\left(\mathrm{O}_{2}{ }^{-}\right)$in the WT and transgenic seedlings under salt stress by histochemical staining with 3,3'-diaminobenzidine (DAB) and photometric nitro blue tetrazolium (NBT). As shown in Figure 4A, under normal growth conditions, there were no differences in $\mathrm{H}_{2} \mathrm{O}_{2}$ and $\mathrm{O}_{2}{ }^{-}$between WT and transgenic lines. Although brown-colored polymeric oxidation products were visualized in all of the salt-stressed plants, transgenic plants exhibited lower accumulations of $\mathrm{H}_{2} \mathrm{O}_{2}$ than WT plants (Figure 4B). 
Enzyme antioxidants play an important role in scavenging reactive oxygen species. Therefore, we assessed the activities of peroxidase (POD), superoxide dismutase (SOD), and catalase (CAT) in transgenic lines and WT. As shown in Figure 4C-E, the activities of three antioxidant enzyme were all up-regulated in all the seedlings with salt treatment, while significantly higher in the transgenic plants than those in WT. The higher activity of SOD, POD, and CAT resulted in lower levels of accumulation of $\mathrm{O}_{2}{ }^{-}$and $\mathrm{H}_{2} \mathrm{O}_{2}$ in transgenic tobacco. RT-qPCR was performed to detect the expression levels of ROS-related genes in the WT and transgenic lines with or without treatment. The results showed that the expression levels of $N b P O D, N b C A T$, and $N b S O D$ in transgenic lines were significantly higher than those in the WT under salt stress (Figure $4 \mathrm{~F}-\mathrm{H}$ ). These results suggested that overexpression of Sm-MIR408 reduced ROS accumulation by activating ROS-related genes and enhancing SOD, POD, and CAT activities under salt stress in tobacco.

A
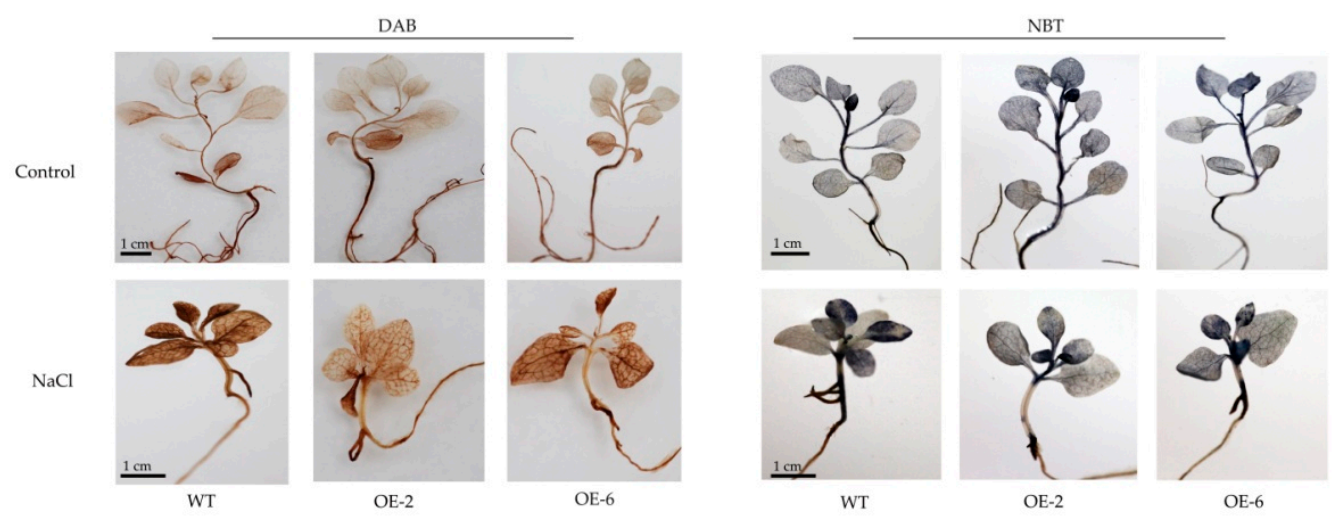

B

C

D

E
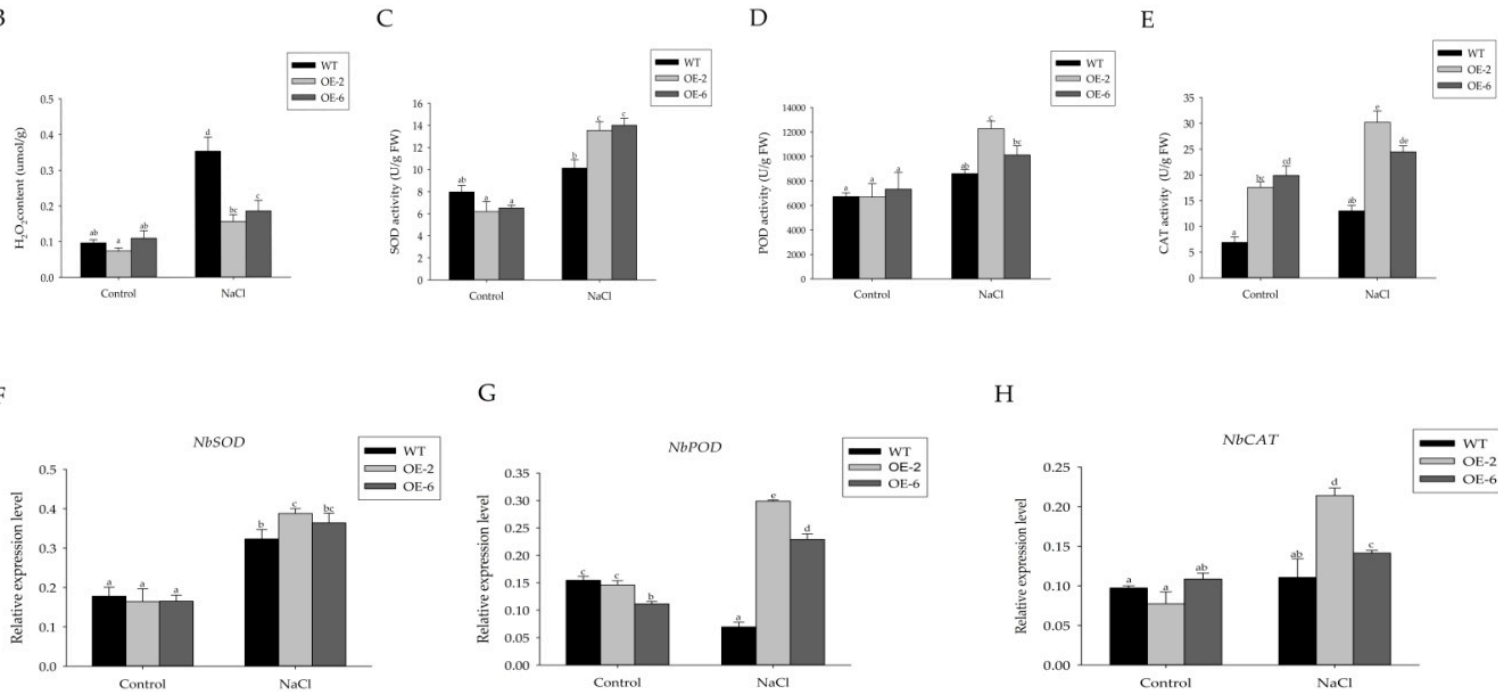

$\mathrm{F}$

G

$\mathrm{H}$
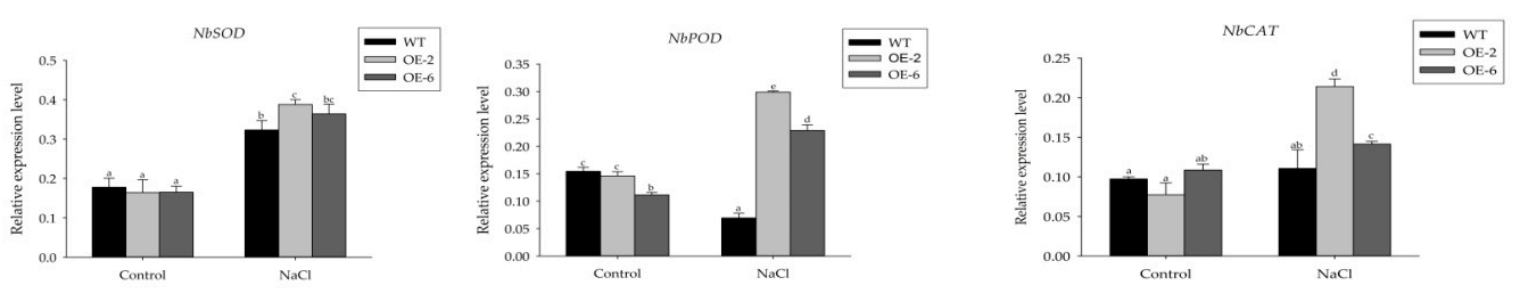

Figure 4. Contents of reactive oxygen species (ROS) in wild type (WT) and miR408-overexpressing Nicotiana benthamiana. (A) 3,3-Diaminobenzidine (DAB) and nitroblue tetrazolium (NBT) staining when three-week-old seedlings were treated with $150 \mathrm{mM} \mathrm{NaCl}$ for 10 days. The strength of color showed the concentration of $\mathrm{H}_{2} \mathrm{O}_{2}$ and $\mathrm{O}_{2}{ }^{-}$. (B) Contents of $\mathrm{H}_{2} \mathrm{O}_{2}$ in tobacco with or without salt treatment. (C-E) The activities of SOD (C), POD (D), and CAT (E) in WT and transgenic lines when three-week-old seedlings were treated with $150 \mathrm{mM} \mathrm{NaCl}$ for 10 days. (F-H) Expression of ROS-responsive genes $\operatorname{NbSOD}(\mathbf{F}), \operatorname{NbPOD}(\mathbf{G})$, and $\operatorname{NbCAT}(\mathbf{H})$, determined via RT-qPCR. The data represent means $\pm \mathrm{SD}$ of three independent experiments. Significant differences were determined using Duncan's multiple range test (indicated by different letters at $p<0.05$ ). 


\section{Discussion}

MicroRNAs (miRNAs) are a class of small non-coding RNAs 20-24 nucleotides in length. Apart from their roles in development, the roles that some of their members play in the complex stress response network have been gradually recognized. For example, overexpressing Osa-miR528 enhances tolerance to nitrogen starvation in creeping bentgrass [36]. Overexpression of miR172a and miR172b in Solanum lycopersicum enhances resistance to Phytophthora infestans [37]. Heterologous expression of a rice miR395 gene in Nicotiana tabacum impairs sulfate homeostasis [38]. Transgenic Agrostis stolonifera overexpressing Osa-miR393a improved multiple stress tolerance, including drought, salt and heat [39]. Transgenic barley that overexpressed hvu-miRX showed drought tolerance [40]. The increasing number of functional studies on plant miRNAs have demonstrated that they are promising candidates for enhancing multiple stress tolerance in plants.

MiR408 is a highly conserved miRNA, and it plays significant roles in plant growth and development. In Brassica napus, the upregulation of miR408 restricted silique development due to inorganic phosphate/copper deficiency [41]. MiR408 regulates vegetative development in Arabidopsis and heading time in wheat [34,42]. The constitutive expression of miR408 can improve photosynthesis, growth, biomass, and seed yield in some plants [43-45]. Recent studies have shown that abiotic stresses induce the aberrant expression of miR408. MiR408 is a typical of miRNAs that respond to the abiotic stress differently depending on the plant species. For example, drought treatment up-regulated miR408 expression in Arabidopsis [22] and Medicago truncatula [21], but down-regulated the expression of miR408 in Oryza sativa [23], Prunus persica [24], and cotton [46]. The expression of miR408 was up-regulated in response to salinity treatment in Arabidopsis [22] and cotton [45], while it was significantly down-regulated in response to salinity stress in rice [47] and radish [48]. Here, we investigated the expression of Sm-MIR408 in response to salt stress. RT-qPCR and GUS staining revealed that miR408 was up-regulated by salinity treatments in S. miltiorrhiza (Figure 1), which was consistent with the expression pattern in Arabidopsis and cotton.

Although the function of miR408 as a key post-transcriptional regulator of gene expression in plant development is well established, its functional response to the environment is very limited. By now, only two documents about the function of miR408 response to abiotic stress have been reported [24,26]. In Arabidopsis, overexpression of miR408 enhances its tolerance to salinity, cold, and oxidative stress, but improved sensitivity to drought and osmotic stress [24]. Transgenic chickpea overexpressing the miR408 increased drought tolerance [26]. These studies hinted that miR408 plays important roles in abiotic stress, but it probably plays the opposite function in different species. Salt stress is one of the main environmental stresses that have a severe effect on the quality and yield of crops. Base on the result that miR408 was up-regulated by salinity treatments in S. miltiorrhiza (Figure 1C,D), the function of the miR408 response to salt stress was carried out in the present study. Transgenic tobacco overexpressing Sm-MIR408 showed higher germination rates and lower growth inhibition than WT under salt stress conditions (Figure 3). Our results indicated that a high expression of miR408 improved tolerance to salinity in tobacco, which agreed with the function of miR408 in Arabidopsis [24].

ROS are important signaling molecules in the regulation of many of biological processes. The accumulation of ROS was enhanced in tissues when plants suffer abiotic stresses. Many studies have revealed that the capacity of ROS scavenging was associated with plant tolerance to abiotic stresses [49-52]. In the present study, all the plants accumulated higher ROS levels under salt stress conditions, while transgenic plants exhibited lower accumulation than WT (Figure 4B). Due to the physical and chemical toxicity of high ROS levels, the ROS-scavenging enzymes, including SOD, POD, and CAT, are activated in plant cells in response to oxidative damage [53]. The low content of ROS is related to the high activity of those enzymes [54]. So, we tested the activity of SOD, POD, and CAT in plants. As shown in Figure 4C-E, the activities of SOD, POD, and CAT were higher in transgenic lines than those in the WT plants under salt stress. However, differences could be observed between the two independent transgenic lines. The relative increase in the CAT and POD activity was higher in OE2 compared to OE6. We speculated that higher expression level of miR408 in OE2 is 
correlated with higher increase of enzyme activity in that line, supporting its involvement in reducing the accumulation of ROS during salinity stress.

MiRNAs play their roles by regulating the expression of target genes. In Populus euphratica, the target PeNAC genes of peu-miR164 were involved in abiotic stress responses [55]. MiR408 is negatively regulated by the availability of copper in Arabidopsis and target genes encoding several copper transporters and a copper chaperone, indicating its central role in the response to copper deficiency [33,34]. Here, RT-qPCR and GUS staining results indicated that Sm-MIR408 in S. miltiorrhiza was strongly induced by copper deficiency, and it was efficiently inhibited by sufficient copper (Figure 1A,B). MiR408 targets genes encoding Copper-transporting ATPase PAA2 and Uclacyanin-2 (UCL-2) in N. benthamiana [35]. Copper-transporting ATPase PAA2 is a copper-transporter that supplies $\mathrm{Cu}$ to plastocyanin (PC), an essential protein for photosynthetic activity in higher plants [56]. Uclacyanin-2 belongs to phytocyanin family, which are blue copper proteins and function as electron transporters [44]. Both targets are related to copper, which is a cofactor of various enzymes, and plays roles in photosynthesis and respiration electron transfer, oxidative stress protection, ethylene perception, and cell wall metabolism [44]. Also, 14 UCLs were regulated under the treatments of abiotic stresses [57]. It was hypothesized that the release of copper from non-essential cuproproteins can act as copper supply buffer [58]. Such a copper release and supply mechanism could be activated during stress responses and supply the copper required for proteins involved in stress responses [26]. We hypothesize that constitutive expression of miR408 resulted in reduced levels of the non-essential cuproprotein Uclacyanin-2, leading to increased availability of copper to those cuproproteins that are required to cope with the stresses, such as the $\mathrm{Cu} / \mathrm{Zn}$ SODs. The connection between the targets of miR408 and antioxidative system or genes involved in abiotic stress response is yet to be understood with greater details.

\section{Materials and Methods}

\subsection{Plant Material and Growth Conditions}

All the plants (including the wild-type S. miltiorrhiza, wild-type N. benthamiana, transgenic S. miltiorrhiza and transgenic $N$. benthamiana) were grown in a greenhouse under standard growth conditions of light and temperature $\left(22^{\circ} \mathrm{C}\right.$, light intensity $100 \mu \mathrm{mol} \mathrm{m}{ }^{-2} \cdot \mathrm{s}^{-1}, 16 / 8 \mathrm{~h} \mathrm{light} /$ dark cycle $)$ with $60 \%$ relative humidity.

The seeds of transgenic tobacco expressing 35S:::MIR408 and WT were cultured vertically on MS medium and MS medium containing $150 \mathrm{mM} \mathrm{NaCl}$ and germination rate was recorded after 7 days. The root length and fresh weight were measured when the seeds were cultured for 21 days under normal and salt stress conditions. Three-week-old transgenic seedlings and WT grown under normal condition were transferred to MS medium or MS medium supplemented with $150 \mathrm{mM} \mathrm{NaCl}$ for 10 days, and harvested for the histochemical detection of ROS, RT-qPCR, the determination of $\mathrm{H}_{2} \mathrm{O}_{2}$ content, and measurements of SOD, POD, and CAT activity. In the experiment, at least 10 seedlings were used for each treatment. All the experiments were performed with three biological replicates.

\subsection{Construction of Plant Expression Vector and Tobacco Transformation}

Amplification of $366 \mathrm{bp}$ miR408 precursor sequences was made from the genomic DNA of S. miltiorrhiza using the primers MIR408-F/R (Table 1), and cloned into the XbaI/SacI sites of pBI121 to generate the Sm-MIR408-overexpressing vector 35S::MIR408. We amplified a 723 bp fragment from the upstream region of Sm-MIR408 with the primers P-MIR408-F/R (Table 1) and cloned it into the PstI/NcoI sites of pCAMBIA-1391Z to obtain the MIR408pro::GUS construct. These recombined vectors were then introduced into N. benthamiana using the Agrobacterium-mediated transformation method. Putative transgenic shoots were selected on agar media containing the presence of $50 \mathrm{mg} / \mathrm{L}$ kanamycin for expressing the 35S::MIR408 construct, and $25 \mathrm{mg}$ /L hygromycin for expressing MIR408pro::GUS construct, and allowed to propagate. T2 generation transgenic tobacco were selected and analyzed. 
Table 1. List of primers used for PCR.

\begin{tabular}{|c|c|}
\hline Primer & Oligo Sequence $5^{\prime}$ to $3^{\prime}$ \\
\hline SmUbi-F & ACССTCACGGGGAAGACCATC \\
\hline SmUbi-R & ACCACGGAGACGGAGGACAAG \\
\hline MIR408-F & ACAGAAAATGGAGGCGAAGAAG \\
\hline MIR408-R & GTCCCTAATCAGTGAGAGACACAGTAA \\
\hline P-MIR408-F & ACGAGCACCGACTCTGATCATTG \\
\hline P-MIR408-R & TCTTCTTCGCCTCCATTT TCTGTAT \\
\hline 35S-F & ACAAAGGCGGCAACAAACG \\
\hline 35S-R & GCCAGTCTTCACGGCGAGT \\
\hline RT-MIR408-F & ACGGGGACGAGACAGAGCAT \\
\hline RT-MIR408-R & GGCTTTCACACCAGCAACATAG \\
\hline NbActin-F & CGTTATGGTTGGAATGGGACAGAA \\
\hline NbActin-R & AAGAACAGGGTGCTCCTCGTGG \\
\hline Copper-transporting ATPase PAA2-F & AAGAGGTCATCGGGGTTAGGA \\
\hline Copper-transporting ATPase PAA2-R & GTAGCGTGAGTTCCACAGCATAAAG \\
\hline Uclacyanin-2-F & ACTTGACGCCTCCGACCACT \\
\hline Uclacyanin-2-R & ACTGCCTCTTCCCTAGACCATG \\
\hline NbSOD-F & GGAGAGCCTTGTCTGATGG \\
\hline NbSOD-R & TGGGTCCTGATTAGCAGTGGT \\
\hline NbPOD-F & GTTGAGAGTTCTTGTCCTGGTGTT \\
\hline NbPOD-R & TATTGGCTCCTCCCTGGTTTG \\
\hline$N b C A T-F$ & CACAGCCACGCTACTCAAGAC \\
\hline$N b C A T-\mathrm{R}$ & CCACCCACCGACGAATAAAG \\
\hline
\end{tabular}

\subsection{Molecular Analysis of Transgenic Tobacco}

To detect whether 35S::MIR408 was integrated into the genome of tobacco, the CaMV35S promoter was amplified by PCR with primers 35S-F/R (Table 1) and genomic DNA extracted from the leaves of kanamycin-resistant tobacco was used as templates.

Total RNA was isolated from the leaves of T2 transgenic lines and WT samples with TRIzol reagent (Invitrogen, CA, USA), and reverse-transcribed into first-strand complementary DNA (cDNA) with PrimeScript ${ }^{\mathrm{TM}}$ RT Master Mix (Takara, Dalian, China), following the manufacturer's protocols. The resultant cDNAs were amplified with RT-MIR408-F/RT-MIR408-R primers (Table 1) to evaluate the transcript level of Sm-MIR408. qRT-PCR was performed on a Roche LightCycler ${ }^{\circledR} 96$ System by using SYBR ${ }^{\circledR}$ Green Premix Ex Taq ${ }^{\mathrm{TM}}$ (TaKaRa). The $N$. benthamiana $\beta$-actin gene was used as the internal control, and amplified with primers NbActin-F/NbActin-R (Table 1).

To determine the level of mature miR408 in N. benthamiana, first-strand cDNA was synthesized using the Mir- $X^{\mathrm{TM}}$ miRNA First-Strand Synthesis Kit (TaKaRa). The miRNA-specific sequences were applied as sense primers, and antisense primers were supplied by the manufacturer. U6 snRNA was used for normalization. The thermal cycling program was as follows: $95^{\circ} \mathrm{C}$ for $60 \mathrm{~s} ; 45$ cycles of $95^{\circ} \mathrm{C}$ for $10 \mathrm{~s}$ and $60^{\circ} \mathrm{C}$ for $30 \mathrm{~s}$; a cycle of $95^{\circ} \mathrm{C}$ for $10 \mathrm{~s}, 65^{\circ} \mathrm{C}$ for $60 \mathrm{~s}$, and $97^{\circ} \mathrm{C}$ for $1 \mathrm{~s}$; and a cycle of $37^{\circ} \mathrm{C}$ for $30 \mathrm{~s}$ to determine the dissociation curves of the amplified products. All reactions were performed with three biological replicates. The primers used for qRT-PCR were listed in Table 1.

\subsection{Copper and Salt Treatment to S. miltiorrhiza and Tobacco}

To analyze how Sm-MIR408 responds to copper, the seeds of $S$. miltiorrhiza were surface-sterilized and germinated on MS medium. Two-week-old uniform S. miltiorrhiza seedlings were transferred to standard MS medium ( $0.1 \mu \mathrm{M}$ copper), MS medium-deficient copper $(0 \mu \mathrm{M}, \mathrm{MS}-\mathrm{Cu})$, and sufficient copper $(5 \mu \mathrm{M}, \mathrm{MS}+\mathrm{Cu})$ for 7 days, followed by RNA isolation and RT-qPCR analysis. The seeds of transgenic tobacco expressing MIR408pro::GUS and WT were surface-sterilized and germinated on MS medium. One-week-old tobacco seedlings were transferred to MS, MS- $\mathrm{Cu}$ and MS+Cu for three weeks, followed by GUS staining. 
For salt treatment, one-month-old S. miltiorrhiza seedlings grown in soil were watered with $150 \mathrm{mM} \mathrm{NaCl}$ for $24 \mathrm{~h}$, and harvested for RT-qPCR analysis, while the control was watered with $\mathrm{ddH}_{2} \mathrm{O}$. The seeds of transgenic tobacco-expressing MIR408pro::GUS and WT were cultured vertically on MS medium and MS medium containing $150 \mathrm{mM} \mathrm{NaCl}$ for three weeks, and followed by GUS staining.

\subsection{GUS Bioassays}

T2 generation transgenic tobacco expressing MIR408pro::GUS were used for analysis. The seeds were germinated on MS medium containing the presence of $25 \mathrm{mg} / \mathrm{L}$ hygromycin. One-month-old transgenic seedlings were transferred to half-strength of Hoagland salt for 2 days. These were subjected to Hoagland salts supplemented with $150 \mathrm{mM} \mathrm{NaCl}$ for $24 \mathrm{~h}$. GUS histochemical staining were performed as described by Jefferson [59].

\subsection{Histochemical Detection of $\mathrm{H}_{2} \mathrm{O}_{2}$ and $\mathrm{O}_{2}{ }^{-}$}

The tobacco seedlings were incubated in NBT solution $(1 \mathrm{mg} / \mathrm{mL}$ in $10 \mathrm{mM}$ phosphate buffer; $\mathrm{pH}$ 7.8) overnight in the dark until blue spots showing the accumulation of $\mathrm{O}_{2}{ }^{-}$appeared. The accumulation of $\mathrm{H}_{2} \mathrm{O}_{2}$ was detected by immersing the plants in a $1 \mathrm{mg} / \mathrm{mL}$ solution of DAB, $\mathrm{pH} 3.8$, overnight. Stained plants were soaked in $95 \%$ ethanol overnight to remove chlorophyll.

\subsection{Determination of $\mathrm{H}_{2} \mathrm{O}_{2}$ Content}

A 100-150 mg sample of fresh tissue was powdered in a mortar together with $1 \mathrm{~mL}$ of frozen acetone and a small amount of quartz sand. The homogenate was centrifuged at $8000 \mathrm{~g}$ for $10 \mathrm{~min}$ at $4{ }^{\circ} \mathrm{C}$, and all the supernatant was taken and set on ice to be tested. The content of $\mathrm{H}_{2} \mathrm{O}_{2}$ was determined following the protocol of the Micro Hydrogen Peroxide Assay Kit (Solarbio, Beijing, China).

\subsection{Measurement of $S O D, P O D$, and CAT Activities}

The seedlings were ground to powder in liquid nitrogen. We added $5 \mathrm{~mL}$ of $50 \mathrm{mmol} / \mathrm{L}$ phosphate-buffered saline containing $1 \% \beta$-mercaptoethanol and $1 \%$ polyvinylpyrrolidone ( $\mathrm{pH} 7.8$ ) to $0.5 \mathrm{~g}$ of sample, and incubated this for $60 \mathrm{~min}$ at $4{ }^{\circ} \mathrm{C}$ After centrifugating for $20 \mathrm{~min}$ with 10,000 rpm at $4{ }^{\circ} \mathrm{C}$, the supernatant (enzyme extraction) was used for analysis. The activities of POD, CAT, and SOD were measured by spectrophotometer utilizing commercial kit (A084-3, A007-1 and A001-4, Jiancheng, Nanjing, China) following the manufacturer's instructions.

\subsection{Expression Profile of ROS-Related Genes and Sm-MIR408 Response to Salt Stress}

We performed RT-qPCR to determine the transcript level of Sm-MIR408 in response to copper and salt treatment. The housekeeping gene SmUbiquitin in S. miltiorrhiza was used as the internal control, and amplified with primers SmUbi-F/SmUbi-R. The N. benthamiana $\beta$-actin gene was used an internal control to quantify the relative expression levels of $N b P O D, N b C A T$ and $N b S O D$ using RT-qPCR assay. To calculate the relative mRNA levels, raw data from RT-qPCR was analyzed using the standard curve method, normalized by the raw data of the internal control.

\section{Conclusions}

We confirmed that the expression of Sm-MIR408 was up-regulated under salt stress and copper starvation in S. miltiorrhiza. Overexpression of Sm-MIR408 in tobacco improved salt tolerance, as manifested by promoted seed germination, reduced levels of reactive oxygen species, increased activities of SOD, POD, and CAT and the expression levels of their genes. MiR408 might serve as a potential target for genetic manipulations to engineer salt stress tolerance in diverse plants.

Author Contributions: Conceptualization, J.N. and X.C.; Investigation, X.G. and J.N.; Methodology, X.G., J.N. and X.C.; Formal analysis, X.G. and X.C.; Writing-Original draft, X.G; Writing-Review \& Editing, X.C.; Project Administration, J.N. and X.C. All authors read and approved the manuscript. 
Funding: This research was funded by the National Natural Science Foundation of China (31870276 and 31670299); the Natural Science Foundation of Shaanxi Province, China (2018JZ3001); the National Key Technologies R \& D Program for Modernization of Traditional Chinese Medicine (2017YFC1701300 and 2017YFC1700706); Major Project of Shaanxi Province, China (2017ZDXM-SF-005); and Fundamental Research Funds for the Central Universities (GK201706004 and GK201703074).

Conflicts of Interest: The authors declare no conflict of interest.

\section{References}

1. Agarwal, P.; Reddy, M.P.; Chikara, J. Wrky: Its structure, evolutionary relationship, DNA-binding selectivity, role in stress tolerance and development of plants. Mol. Boil. Rep. 2011, 38, 3883-3896. [CrossRef]

2. Yujia, L.; Xiaoyu, J.; Xianguang, N.; Min, Q.; Lei, Z.; Zilong, T.; Huimin, Z.; Lin, H.; Shengnan, L.; Bing, Z. Arabidopsis AtbHLH112 regulates the expression of genes involved in abiotic stress tolerance by binding to their E-box and GCG-box motifs. New Phytol. 2015, 207, 692-709.

3. Altman, A. Recent advances in engineering plant tolerance to abiotic stress: Achievements and limitations. Curr. Opin. Biotechnol. 2005, 16, 123-132.

4. Zhu, J.K. Salt and drought stress signal transduction in plants. Annu. Rev. Plant Boil. 2002, 53, $247-273$. [CrossRef]

5. Mittler, R.; Vanderauwera, S.; Gollery, M.; Van, B.F. Reactive oxygen gene network of plants. Trends Plant Sci. 2004, 9, 490-498. [CrossRef]

6. Voinnet, O. Origin, biogenesis, and activity of plant micrornas. Cell 2009, 136, 669-687. [CrossRef]

7. Chen, K.; Rajewsky, N. The evolution of gene regulation by transcription factors and microRNAs. Nat. Rev. Genet. 2007, 8, 93-103. [CrossRef]

8. Hobert, O. Gene regulation by transcription factors and microRNAs. Science 2008, 319, 1785-1786. [CrossRef]

9. Zhang, B. MicroRNA: A new target for improving plant tolerance to abiotic stress. J. Exp. Bot. 2015, 66, 1749-1761. [CrossRef]

10. Palatnik, J.F.; Allen, E.; Wu, X.; Schommer, C.; Schwab, R.; Carrington, J.C.; Weigel, D. Control of leaf morphogenesis by microRNAs. Nature 2003, 425, 257-263. [CrossRef]

11. Lauter, N.; Kampani, A.; Carlson, S.; Goebel, M.; Moose, S.P. microRNA172 down-regulates glossy15 to promote vegetative phase change in maize. Proc. Natl. Acad. Sci. USA 2005, 102, 9412-9417. [CrossRef]

12. Yu, L.; Yu, X.; Shen, R.; He, Y. HYL1 gene maintains venation and polarity of leaves. Planta 2005, 221, $231-242$. [CrossRef]

13. Wu, G.; Park, M.Y.; Conway, S.R.; Wang, J.W.; Weigel, D.; Poethig, R.S. The sequential action of miR156 and miR172 regulates developmental timing in Arabidopsis. Cell 2009, 138, 750-759. [CrossRef]

14. Zhao, L.; Kim, Y.; Dinh, T.T.; Chen, X. miR172 regulates stem cell fate and defines the inner boundary of APETALA3 and PISTILLATA expression domain in Arabidopsis floral meristems. Plant J. 2010, 51, 840-849. [CrossRef]

15. Tao, W.; Sun, M.Y.; Wang, X.S.; Li, W.B.; Li, Y.G. Over-expression of GmGla-regulated soybean miR172a confers early flowering in transgenic Arabidopsis thaliana. Int. J. Mol. Sci. 2016, 17, 645.

16. Reyes, J.L.; Chua, N.H. ABA induction of miR159 controls transcript levels of two MYB factors during Arabidopsis seed germination. Plant J. 2010, 49, 592-606. [CrossRef]

17. Zhou, M.; Li, D.; Li, Z.; Hu, Q.; Yang, C.; Zhu, L.; Luo, H. Constitutive expression of a miR319 gene alters plant development and enhances salt and drought tolerance in transgenic creeping bentgrass. Plant Signal. Behav. 2014, 161, 1375-1391. [CrossRef]

18. Sunkar, R.; Kapoor, A.; Zhu, J.K. Posttranscriptional induction of two Cu/Zn superoxide dismutase genes in Arabidopsis is mediated by downregulation of miR398 and important for oxidative stress tolerance. Plant Cell 2006, 18, 2051-2065. [CrossRef]

19. Zhao, Q.; Nakashima, J.; Chen, F.; Yin, Y.; Fu, C.; Yun, J.; Shao, H.; Wang, X.; Wang, Z.Y.; Dixon, R.A. Laccase is necessary and nonredundant with peroxidase for lignin polymerization during vascular development in Arabidopsis. Plant Cell 2013, 25, 3976-3987. [CrossRef]

20. Schuetz, M.; Benske, A.; Smith, R.A.; Watanabe, Y.; Tobimatsu, Y.; Ralph, J.; Demura, T.; Ellis, B.; Samuels, A.L. Laccases direct lignification in the discrete secondary cell wall domains of protoxylem. Plant Physiol. 2014, 166, 798-807. [CrossRef] 
21. Trindade, I.; Capitão, C.; Dalmay, T.; Fevereiro, M.P.; Santos, D.M.D. miR398 and miR408 are up-regulated in response to water deficit in Medicago truncatula. Planta 2010, 231, 705-716. [CrossRef]

22. Barciszewskapacak, M.; Milanowska, K.; Knop, K.; Bielewicz, D.; Nuc, P.; Plewka, P.; Pacak, A.M.; Vazquez, F.; Karlowski, W.; Jarmolowski, A. Arabidopsis microRNA expression regulation in a wide range of abiotic stress responses. Front. Plant Sci. 2015, 6, 410.

23. Zhou, L.; Liu, Y.; Liu, Z.; Kong, D.; Duan, M.; Luo, L. Genome-wide identification and analysis of drought-responsive micrornas in Oryza sativa. J. Exp. Bot. 2010, 61, 4157-4168. [CrossRef]

24. Eldem, V.; Akcay, U.C.; Ozhuner, E.; Bakır, Y.; Uranbey, S.; Unver, T. Genome-wide identification of miRNAs responsive to drought in peach (Prunus persica) by high-throughput deep sequencing. PLoS ONE 2012, 7, e50298. [CrossRef]

25. Zhang, H.; Zhao, X.; Li, J.; Cai, H.; Deng, X.W.; Li, L. microRNA408 is critical for the HY5-SPL7 gene network that mediates the coordinated response to light and copper. Plant Cell 2014, 26, 4933-4953. [CrossRef]

26. Ma, C.; Burd, S.; Lers, A. MiR408 is involved in abiotic stress responses in Arabidopsis. Plant J. 2015, 84, 169-187. [CrossRef]

27. Hajyzadeh, M.; Turktas, M.; Khawar, K.M.; Unver, T. MiR408 overexpression causes increased drought tolerance in chickpea. Gene 2015, 555, 186-193. [CrossRef]

28. Committee for the Pharmacopoeia of People's Republic of China. Pharmacopoeia of P.R. China; Part 1; Chemical Industry Publishing House: Biejing, China, 2015; p. 76.

29. Hao, P.P.; Jiang, F.; Chen, Y.G.; Yang, J.; Zhang, K.; Zhang, M.X.; Zhang, C.; Zhao, Y.X.; Zhang, Y. Evidence for traditional chinese medication to treat cardiovascular disease. Nat. Rev. Cardiol. 2015, 12, 374. [CrossRef]

30. Xu, X.; Jiang, Q.; Ma, X.; Ying, Q.; Shen, B.; Qian, Y.; Song, H.; Wang, H. Deep sequencing identifies tissue-specific microRNAs and their target genes involving in the biosynthesis of tanshinones in Salvia miltiorrhiza. PLoS ONE 2014, 9, e111679. [CrossRef]

31. Zhang, L.; Wu, B.; Zhao, D.; Li, C.; Shao, F.; Lu, S. Genome-wide analysis and molecular dissection of the SPL gene family in Salvia miltiorrhiza. J. Integr. Plant Biol. 2014, 56, 38-50. [CrossRef]

32. Guo, X.R.; Yang, X.B.; Wang, H.Q.; Ming, F.L.; She, X.; Cao, X.Y. Cloning and expression analysis of miR408 precursor sequences from Salvia miltiorrhiza. Plant Sci. J. 2016, 34, 430-438.

33. Abdel-Ghany, S.E.; Pilon, M. MicroRNA-mediated systemic down-regulation of copper protein expression in response to low copper availability in Arabidopsis. J. Biol. Chem. 2008, 283, 15932-15945. [CrossRef]

34. Zhang, H.; Li, L. SQUAMOSA promoter binding protein-like7 regulated microRNA408 is required for vegetative development in Arabidopsis. Plant J. 2013, 74, 98-109. [CrossRef]

35. Baksa, I.; Nagy, T.; Barta, E.; Havelda, Z.; Várallyay, É.; Silhavy, D.; Burgyán, J.; Szittya, G. Identification of Nicotiana benthamiana microRNAs and their targets using high throughput sequencing and degradome analysis. BMC Genom. 2015, 16, 1025. [CrossRef]

36. Yuan, S.; Li, Z.; Li, D.; Yuan, N.; Hu, Q.; Luo, H. Constitutive expression of rice microRNA528 alters plant development and enhances tolerance to salinity stress and nitrogen starvation in creeping bentgrass. Plant Physiol. 2015, 169, 576-593. [CrossRef]

37. Luan, Y.; Cui, J.; Li, J.; Jiang, N.; Liu, P.; Meng, J. Effective enhancement of resistance to phytophthora infestans by overexpression of miR172a and b in Solanum lycopersicum. Planta 2017, 247, 127-138. [CrossRef]

38. Yuan, N.; Yuan, S.; Li, Z.; Li, D.; Hu, Q.; Luo, H. Heterologous expression of a rice miR395 gene in Nicotiana tabacum impairs sulfate homeostasis. Sci. Rep. 2016, 6, 28791. [CrossRef]

39. Zhao, J.; Yuan, S.; Zhou, M.; Yuan, N.; Li, Z.; Hu, Q.; Bethea, J.F.; Liu, H.; Li, S.; Luo, H. Transgenic creeping bentgrass overexpressing osa-miR393a exhibits altered plant development and improved multiple stress tolerance. Plant Biotechnol. J. 2018. [CrossRef]

40. Zhou, H.; Hussain, S.S.; Hackenberg, M.; Bazanova, N.; Eini, O.; Li, J.; Gustafson, P.; Shi, B. Identification and characterisation of a previously unknown drought tolerance-associated microRNA in barley. Plant J. 2018, 95, 138-149. [CrossRef]

41. Chen, L.; Zhang, X.; Liu, T.; Niu, S.; Wen, J.; Yi, B.; Ma, C.; Tu, J.; Fu, T. Identification of miRNAs that regulate silique development in Brassica napus. Plant Sci. 2018, 269, 106-117. [CrossRef]

42. Zhao, X.; Hong, P.; Wu, J.; Chen, X.; Ye, X.; Pan, Y.; Wang, J.; Zhang, X. The tae-miR408-mediated control of TaTOC1 genes transcription is required for the regulation of heading time in wheat. Plant Physiol. 2016, 170, 1578. [CrossRef] 
43. Pan, J.; Huang, D.; Guo, Z.; Kuang, Z.; Zhang, H.; Xie, X.; Ma, Z.; Gao, S.; Lerdau, M.T.; Chu, C. Overexpression of microRNA408 enhances photosynthesis, growth, and seed yield in diverse plants. J. Integr. Plant Biol. 2018, 60, 323-340. [CrossRef]

44. Zhang, J.P.; Yu, Y.; Feng, Y.Z.; Zhou, Y.F.; Zhang, F.; Yang, Y.W.; Lei, M.Q.; Zhang, Y.C.; Chen, Y.Q. miR408 regulates grain yield and photosynthesis via a phytocyanin protein. Plant Physiol. 2017, 175, 1175. [CrossRef]

45. Song, Z.; Zhang, L.; Wang, Y.; Li, H.; Li, S.; Zhao, H.; Zhang, H. Constitutive expression of miR408 improves biomass and seed yield in Arabidopsis. Front. Plant Sci. 2017, 8, 2114. [CrossRef]

46. Xie, F.; Wang, Q.; Sun, R.; Zhang, B. Deep sequencing reveals important roles of microRNAs in response to drought and salinity stress in cotton. J. Exp. Bot. 2015, 66, 789-804. [CrossRef]

47. Anca, M.; Narendra, T. microRNAs targeting DEAD-box helicases are involved in salinity stress response in rice (Oryza sativa L.). BMC Plant Biol. 2012, 12, 183.

48. Sun, X.; Liang, X.; Yan, W.; Yu, R.; Zhu, X.; Luo, X.; Gong, Y.; Wang, R.; Limera, C.; Zhang, K. Identification of novel and salt-responsive miRNAs to explore miRNA-mediated regulatory network of salt stress response in radish (Raphanus sativus L.). BMC Genom. 2015, 16, 197. [CrossRef]

49. Wang, C.; Lu, G.; Hao, Y.; Guo, H.; Guo, Y.; Zhao, J.; Cheng, H. ABP9, a maize bZIP transcription factor, enhances tolerance to salt and drought in transgenic cotton. Planta 2017, 246, 453-469. [CrossRef]

50. Taheri, P.; Kakooee, T. Reactive oxygen species accumulation and homeostasis are involved in plant immunity to an opportunistic fungal pathogen. J. Plant Physiol. 2017, 216, 152-163. [CrossRef]

51. Yujie, F.; Kaifeng, L.; Hao, D.; Yan, X.; Huazhi, S.; Xianghua, L.; Lizhong, X. A stress-responsive NAC transcription factor SNAC3 confers heat and drought tolerance through modulation of reactive oxygen species in rice. J. Exp. Bot. 2015, 66, 6803-6817.

52. Swain, D.M.; Sahoo, R.K.; Srivastava, V.K.; Tripathy, B.C.; Tuteja, R.; Tuteja, N. Function of heterotrimeric G-protein $\gamma$ subunit RGG1 in providing salinity stress tolerance in rice by elevating detoxification of ROS. Planta 2016, 245, 367-383. [CrossRef]

53. Mittler, R. Abiotic stress, the field environment and stress combination. Trends Plant Sci. 2006, 11, 15-19. [CrossRef]

54. Zhang, S.; Zhuang, K.; Wang, S.; Lv, J.; Ma, N.N.; Meng, Q. A novel tomato SUME E3 ligase, SISIZ1, confers drought tolerance in transgenic tobacco. J. Integr. Plant Biol. 2017, 59, 102-117. [CrossRef]

55. Lu, X.; Dun, H.; Lian, C.; Zhang, X.; Yin, W.; Xia, X. The role of peu-miR164 and its target PeNAC genes in response to abiotic stress in Populus euphratica. Plant Physiol. Biochem. 2017, 115, 418-438. [CrossRef]

56. Tapken, W.; Ravet, K.; Pilon, M. Plastocyanin controls the stabilization of the thylakoid Cu-transporting P-type ATPase PAA2/HMA8 in response to low copper in Arabidopsis. J. Biol. Chem. 2012, 287, 18544-18550. [CrossRef]

57. Ma, H.; Zhao, H.; Liu, Z.; Zhao, J. The phytocyanin gene family in rice (Oryza sativa L.): Genome-wide identification, classification and transcriptional analysis. PLoS ONE 2011, 6, e25184. [CrossRef]

58. Ravet, K.; Pilon, M. Copper and iron homeostasis in plants: The challenges of oxidative stress. Antioxid. Redox Signal. 2013, 19, 919-932. [CrossRef]

59. Jefferson, R.A.; Kavanagh, T.A.; Bevan, M.W. GUS fusions: Beta-glucuronidase as a sensitive and versatile gene fusion marker in higher plants. EMBO J. 1987, 6, 3901-3907. [CrossRef]

(C) 2018 by the authors. Licensee MDPI, Basel, Switzerland. This article is an open access article distributed under the terms and conditions of the Creative Commons Attribution (CC BY) license (http://creativecommons.org/licenses/by/4.0/). 